\title{
Biochemistry of Selenium
}




\title{
BIOCHEMISTRY OF THE ELEMENTS
}

\author{
Series Editor: Earl Frieden \\ Florida State University \\ Tallahassee, Florida
}

\section{Volume 1 BIOCHEMISTRY OF NONHEME IRON Anatoly Bezkorovainy \\ Volume 2 BIOCHEMISTRY OF SELENIUM \\ Raymond J. Shamberger}

A Continuation Order Plan is available for this series. A continuation order will bring delivery of each new volume immediately upon publication. Volumes are billed only upon actual shipment. For further information please contact the publisher. 


\section{Biochemistry of Selenium}

\section{Raymond J. Shamberger}

The Cleveland Clinic Foundation

Cleveland, Ohio 


\section{Library of Congress Cataloging in Publication Data}

Shamberger, Raymond J., 1934-

Biochemistry of selenium.

(Biochemistry of the elements, v. 2)

Bibliography: $p$.

Includes index.

1. Selenium - Physiological effect. 2. Selenium-Metabolism. I. Title. II. Series. [DNLM: 1. Selenium. QU 130 B6144 1980 v. 2]

QP535.S5S45 $1983 \quad 574.19 / 214$

$82-22316$

ISBN-13: 978-1-4684-4315-8 e-ISBN-13: 978-1-4684-4313-4

DOI: $10.1007 / 978-1-4684-4313-4$

(C) 1983 Plenum Press, New York

Softcover reprint of the hardcover 1st edition 1983

A Division of Plenum Publishing Corporation

233 Spring Street, New York, N.Y. 10013

All rights reserved

No part of this book may be reproduced, stored in a retrieval system, or transmitted in any form or by any means, electronic, mechanical, photocopying, microfilming, recording, or otherwise, without written permission from the Publisher 


\section{Preface}

In recent years many exciting research results have indicated that selenium, depending on its concentration, can influence mammalian metabolism. It has been estimated that in selenium-deficient areas, selenium or selenium-vitamin E combinations added to animal feed can prevent annual losses to beef and dairy cattle and sheep valued at 545 million dollars and poultry and swine losses valued at 82 million dollars.

Some animal diseases that can be prevented by a selenium-supplemented diet include liver necrosis, nutritional muscular dystrophy, exudative diathesis, pancreatic degeneration, mulberry heart disease, infertility, growth impairment, periodontal disease, and encephalomalacia. Selenium intake levels are dependent on the plant or animal feed concentrations, which, in turn, are dependent on the $\mathrm{pH}$ of the soil and the types of rocks from which the soils are derived.

At normal metabolic levels selenium possesses an antioxidant affect manifested through glutathione peroxidase, and selenium also has an effect on cytochrome P-450 and heme metabolism. Comparisons are made between metabolism of selenium and sulfur in plants, animals, and humans. At greater selenium intake levels acute poisoning occurs when high-selenium-content (10,000 ppm Se) plants are consumed in large quantities. The toxic reactions were first manifested in cavalry horses near Fort Randall, Nebraska, in the 1860s.

Selenium has been found to be a potent anticarcinogen for a variety of chemically-induced cancers, and some inverse relationships between selenium occurrence and human cancer mortality have been demonstrated. The anticarcinogenicity of selenium holds much promise in regard to finding the body's anticancer mechanism. If this mechanism is elucidated, perhaps cancer control can be achieved. The potential of selenium may even surpass that of interferon. In addition, selenium has been shown to prevent Keshan disease, a severe myocardiopathy which occurs in young Chinese children. Inverse epidemiological relationships have also been observed between coronary heart disease and environmental selenium. 
Much evidence indicates that selenium or selenium-vitamin E combinations counteract the toxic effects of mercury, methylmercury, cadmium, lead, silver, thallium, and arsenic in animals. Selenium-vitamin E combinations may offer a workable antidote to metal toxicity in humans exposed to these environmental hazards.

The forms of naturally occurring selenium are classified according to whether they are of low or high molecular weight. The synthetic forms of selenium along with their chemotherapeutic effects are also outlined. Because the levels of selenium are important in tissues, numerous nondestructive as well as destructive methods of selenium analyses are outlined.

In order to reduce the cost of the textbook, lists of several references were reduced to the most recent reference. The author wishes to thank Kathryn Risko, Phyllis Pittman, and Helen Brewster for their excellent typing, and Barbara DeWitt, Cindy Kopf, Ann McHugh, and Andrea Yartin for their careful help in proofreading. 


\section{Contents}

\section{Forms of Selenium}

1.1 Low Molecular-Weight Compounds . . . . . . . . 1

1.1.1 Selenocysteine $\ldots \ldots \ldots \ldots \ldots \ldots \ldots \ldots \ldots$. 1

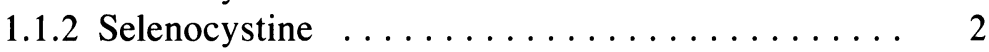

1.1.3 Selenohomocystine ................ 2

1.1.4 Se-methylselenocysteine $\ldots \ldots \ldots \ldots \ldots \ldots \ldots 3$

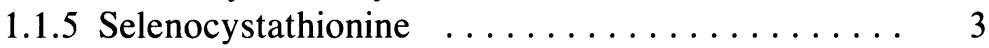

1.1.6 Selenomethionine ................ 4

1.1.7 Se-methylselenomethionine $\ldots \ldots \ldots \ldots \ldots \ldots$. 5

1.1.8 Dimethyl Selenide . . . . . . . . . . . . . . 6

1.1.9 Dimethyl Diselenide ................. 6

1.1.10 Trimethyl Selenonium ............... 6

1.1.11 Elemental Selenium ............... 7

1.1.12 Selenotaurine .................. 7

1.1.13 Selenocoenzyme A . . . . . . . . . . . 7

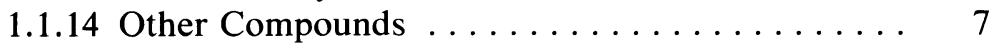

1.2 Macromolecular Forms of Selenium . . . . . . . . . . 8

1.2.1 Formate Dehydrogenase $\ldots \ldots \ldots \ldots \ldots \ldots .8$

1.2.2 Glycine Reductase ................ 11

1.2.3 Nicotinic Acid Hydroxylase . . . . . . . . . . . 15

1.2.4 Xanthine Hydrogenase . . . . . . . . . . . . . 16

1.2 .5 Thiolase ..................... 16

1.2.6 Glutathione Peroxidase .............. 17

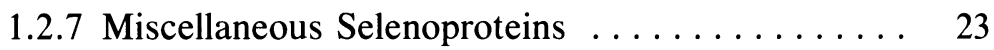

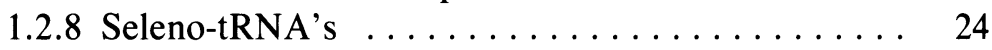

References ........................ 25

2. Selenium Deficiency Diseases in Animals

2.1 Introduction $\ldots \ldots \ldots \ldots \ldots \ldots \ldots \ldots \ldots \ldots \ldots \ldots \ldots$

2.2 Dietary Liver Necrosis and Factor $3 \ldots \ldots \ldots \ldots . \ldots 32$

2.2.1 Discovery ................... 32 
2.2.2 Pathology .................... 36

2.2.3 Biochemical Defect ................ 37

2.2.4 Hepatosis Dietetica ................. 38

2.3 Nutritional Muscular Dystrophy $\ldots \ldots \ldots \ldots \ldots \ldots .38$

2.3.1 Pathology .................... 38

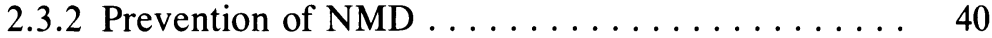

2.4 Exudative Diathesis .................. 44

2.5 Pancreatic Degeneration .................. 45

2.6 Mulberry Heart Disease . . . . . . . . . . . . . . . . 46

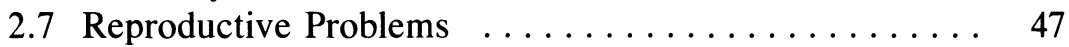

2.8 Myopathy of the Gizzard $\ldots \ldots \ldots \ldots \ldots \ldots \ldots \ldots . \ldots$

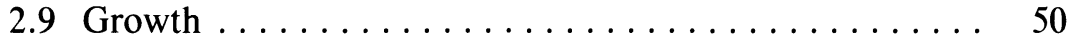

2.10 Selenium-Responsive Unthriftiness of Sheep and Cattle ......................... 51

2.11 Periodontal Disease of Ewes $\ldots \ldots \ldots \ldots \ldots \ldots . \ldots 2$

2.12 Encephalomalacia .................. 52

References........................ 54

3. Metabolism of Selenium

3.1 Absorption ...................... 59

3.2 Placental Transfer ..................... 65

3.3 Mechanism of the Antioxidant Action of Selenium ..... 65

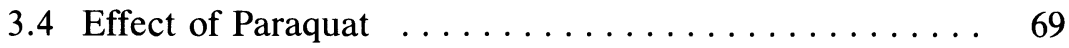

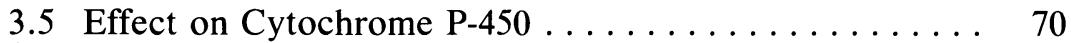

3.6 Selenium and Hepatic Heme Metabolism .......... 71

References .......................... 72

4. Comparative Metabolism and Biochemistry of Selenium and Sulphur

4.1 Introduction . . . . . . . . . . . . . . . 77

4.2 Comparative Metabolism of Selenium and Sulphur ... 78

4.2.1 Microorganisms .................. 78

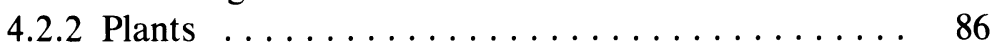

4.2 .3 Animals ...................... 95

4.3 Comparative Biochemistry of Selenium and Sulphur ... 106

4.3.1 Selenopersulfide as an Electron Transfer Catalyst . 106

4.3.2 Iron-Sulphur Proteins ................ 110

4.3.3 Sulphur Salts and Selenium Toxicity in Animals .. 111

4.3.4 Other Selenium-Sulphur Interactions ........ 114

References ......................... 116 
5. Biological Interactions of Selenium with Other Substances

5.1 Cadmium ........................ 125

5.1.1 Pathological Effects ................. 125

5.1 .2 Cadmium-Zinc Interactions $\ldots \ldots \ldots \ldots \ldots . . \ldots 127$

5.1.3 Cadmium-Selenium Interactions . . . . . . . . . . 129

5.1.4 Effect on Drug Response . . . . . . . . . . . . 134

5.2 Arsenic .......................... 135

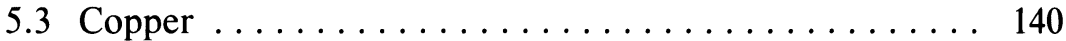

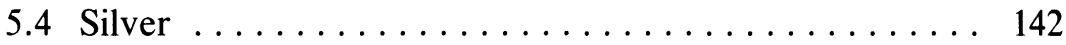

5.5 Cobalt ......................... 144

5.6 Manganese ......................... 146

5.7 Lead . . . . . . . . . . . . . . . . . . . . . . 147

5.8 Mercury . . . . . . . . . . . . . . . . . . . . . 149

5.8.1 Inorganic and Organic Mercury . . . . . . . . . . 149

5.8.2 Tissue Distribution ................ 151

5.8.3 Properties of the Mercury-Selenium Complex ... 152

5.8 .4 Teratogenicity . . . . . . . . . . . . . 152

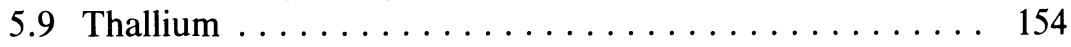

5.10 Tellurium ....................... 154

5.11 Vanadium ....................... 155

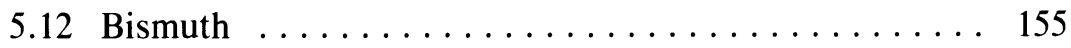

5.13 Other Substances ................... 156

References........................ 157

6. Environmental Occurrence of Selenium

6.1 Geochemistry of Selenium . . . . . . . . . . . . . . 167

6.2 Soil Selenium .................... 170

6.3 Uptake and Concentration of Trace Elements in the

Roots, Stems, and Leaves of Plants . . . . . . . . . 171

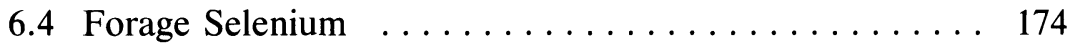

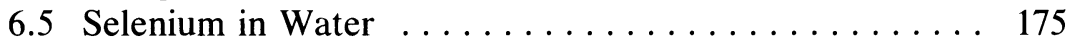

6.6 Selenium in Food ...................... 175

6.7 Intakes and Recommended Daily Allowance in Humans 180

6.8 Regulations in Regard to Animal Diets . . . . . . . . . . 180

References ........................ 181

\section{Toxicity of Selenium}

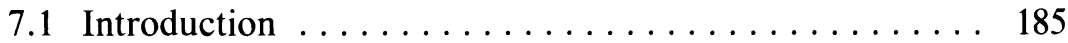

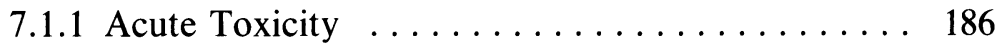

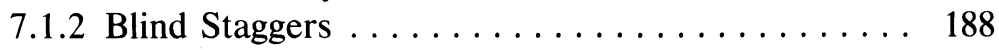


7.1.3 Alkalai Disease ................... 188

7.1 .4 Toxicity in Rabbits . . . . . . . . . . . . . 189

7.1.5 Toxicity in Hamsters . . . . . . . . . . . . . . . . . 190

7.1.6 Toxicity in Sheep . . . . . . . . . . . . . . . 190

7.1.7 Toxicity in Rats . . . . . . . . . . . . . . . . . 191

7.1.8 Effect of Diet on Toxicity . . . . . . . . . . . . . 192

7.1.9 Biochemical Lesions . . . . . . . . . . . . . . 195

7.1.10 $\mathrm{LD}_{50}$ of Various Selenium Compounds ...... 197

7.2 Industrial Medical Aspects . . . . . . . . . . . . . 201

7.2.1 Occupational Hazards . . . . . . . . . . . 201

7.2.2 Permissible Limits for Selenium Exposure . . . . . 202

7.2 .3 Toxicity in Humans . . . . . . . . . . . . . 202

References .......................... 204

8. Selenium in Health and Disease

8.1 Selenium and Cancer . . . . . . . . . . . . . . . 207

8.1.1 Skin Cancer . . . . . . . . . . . . . . . . . . . 207

8.1 .2 Liver Cancer . . . . . . . . . . . . . . . . . . . . 209

8.1 .3 Colon Cancer . . . . . . . . . . . . . . . . 212

8.1 .4 Breast Cancer ..................... 214

8.1.5 Tracheal Cancer ................. 222

8.1.6 Chemotherapeutic Effect of Selenium . . . . . . 222

8.1.7 Epidemiological Relationship ............ 225

8.1.8 Selenium Blood Levels in Cancer Patients . . . . . 227

8.1.9 Selenium as a Carcinogen . . . . . . . . . . . . 229

8.2 Selenium and Mutagenesis . . . . . . . . . . . . 232

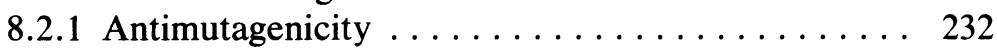

8.2 .2 Mutagenicity ................... 235

8.3 Selenium and Immunity . . . . . . . . . . . . . . 239

8.3.1 Effect of Selenium on Humoral Immunity . . . . . 239

8.3.2 Cell-Mediated Immunity . . . . . . . . . . . . 241

8.3.3 Nonspecific Immune Effects of Selenium ...... 242

8.4 Selenium and Dental Caries ................ 243

8.5 The Anti-Inflammatory Properties of Selenium ....... 246

8.6 Selenium and Heart Disease . . . . . . . . . . . . . . 247

8.6.1 Animals . . . . . . . . . . . . . . . . . . . 247

8.6.2 Humans ..................... 250

8.7 Selenium and Aging $\ldots \ldots \ldots \ldots \ldots \ldots \ldots \ldots \ldots \ldots \ldots$

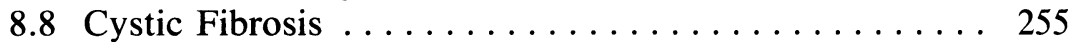

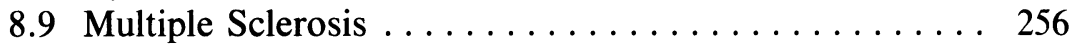

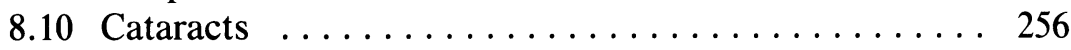

8.11 Other Diseases ........................ 257 
8.12 Radioselenium as a Diagnostic Agent .......... 258

References ........................... 259

9. Synthetic Forms of Selenium and Their Chemotherapeutic Uses

9.1 Anti-Infective Agents . . . . . . . . . . . . . . . 273

9.1.1 Antibacterial . . . . . . . . . . . . . . 273

9.1.2 Antiviral .................... 276

9.2 Antifungal Agents . . . . . . . . . . . . . . . . . 277

9.3 Antiparasitic Agents . . . . . . . . . . . . . . . . 279

9.4 Compounds Affecting the Central Nervous System . . . 280

9.4.1 Hypnotics . . . . . . . . . . . . . . . . . 280

9.4.2 Analgesics and Local Anesthetics .......... 281

9.4.3 Tranquilizing Drugs ................. 282

9.5 Compounds that Affect the Autonomic Nervous System 284

9.6 Compounds that Affect the Circulatory System . . . . . 287

9.7 Anti-Inflammatory Compounds . . . . . . . . . . . . 287

9.8 Antihistamines . . . . . . . . . . . . . . . . 288

9.9 Anticancer Agents . . . . . . . . . . . . . . . . 289

9.10 Antiradiation Agents . . . . . . . . . . . . . . . . . . . 294

9.11 Steroids . . . . . . . . . . . . . . . . . . . . 297

9.12 Selenocoenzyme A . . . . . . . . . . . . . . . . . 299

9.13 Selenium-Containing Carbohydrates . . . . . . . . 300

9.14 Seleno-Amino Acids . . . . . . . . . . . . . . . . 301

References ............................ 304

10. Analytical Methods of Selenium Determination

10.1 Introduction $\ldots \ldots \ldots \ldots \ldots \ldots \ldots \ldots \ldots \ldots \ldots \ldots \ldots$

10.2 Sample Preparation and Storage . . . . . . . . . . 311

10.3 Destructive Analysis . . . . . . . . . . . . 312

10.3.1 Ashing ................... 313

10.3.2 Closed-System Combustion . . . . . . . . . . 313

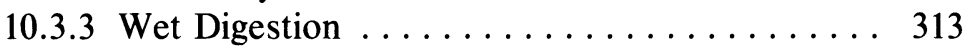

10.3.4 Measurement of Selenium . . . . . . . . . . 314

10.4 Nondestructive Analysis . . . . . . . . . . . . . . 321

10.4.1 Neutron Activation Analysis . . . . . . . . . 321

10.4.2 X-Ray Fluorescence Analysis . . . . . . . . . 322

10.4.3 Proton-Induced X-Ray Emission . . . . . . . . 323

References ....................... 323

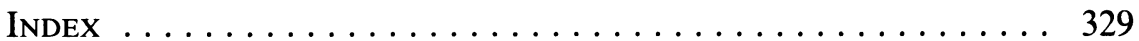

